\title{
Control System Design of Automatic Door Based on PLC
}

\author{
Yangyang $\mathrm{Xu}^{\mathrm{a}}$, Ying Wang ${ }^{\mathrm{b}}$ \\ Zhengzhou University of Industrial Technology, Zhengzhou 450007, China \\ axuyangyang0120@126.com, bWin0725@163.com
}

Keywords: PLC, S7-200, automatic door, control system.

\begin{abstract}
Aiming at enhancing the reliability of the automatic door operation S7-200 programmable logic controller (PLC) was used as the core of the automatic door system. The principle of control system was analyzed with description of the control system hardware it was introduced that the working process of control circuits about human body detection sensor and automatic door position detection and obstacle detection and so on. It was introduced how to choose a PLC type to really settle the I/O orders Combining the operation characteristics of programmable controller a reasonable optimization was made for the work flow of the system. On this basis using structured programming method the program flow charts of main program module and opening door subroutine module and closing door subroutine module were developed. The debugging of the control system was developed and the control system has been adopted. The practical application shays prat the control system is good and reliable.
\end{abstract}

\section{Introduction}

PLC is a control device that specifically designed for industrial automatic control, it has been widely used in the field of industrial automation, and it has the characteristics of strong anti-interference ability, high reliability small size, design, use and easy maintenance and so on. The focus of the study is how to use S7-200 series PLC to realize the control system design of automatic door based on PLC.

\section{Working Principle}

The system uses PLC as the main controller of the automatic door. When the induction detector to detect someone close, the pulse signal is transmitted to PLC, PLC judges after the notice of the motor running, while monitoring the motor rpm to inform the motor at a certain point in time acceleration and running into the slow, motor running forward drive door open. When the door is opened, it is judged by PLC and remains open until there is no one on the door. If there is no one on the door, PLC notify the motor as a reverse movement, in a short time to automatically close the door[1]. In the closing process, if someone close to the probe, PLC automatically switch to the state of the door.

\section{Hardware Components}

\subsection{Human Body Induction Detector.}

The human body induction detection circuit is composed of a passive infrared probe, amplifying circuit and electronic switch circuit, which are arranged on both sides of the automatic door. The human body induction detector needs 2 , respectively used to feel inside and outside of the door, 
take up PLC two input points.

\subsection{Automatic Door Operation Position Detection.}

Two travel switches are provided for this purpose, a position which is used for detecting the door fully opened, the door opening limit switch is used for detecting the position when the door is closed completely. When the door is in the process of operation, close to the maximum position, the limit switch signal Passed to PLC, the motor stops running. When the door is close to the limit switch, the motor runs at low speed, and the motor is controlled by the program.

\subsection{Stepping Motor Drive.}

There are two ways to control the motor in PLC: one is the pulse + direction control; the one is the positive and negative to the pulse output. First methods are used here. PLC high speed pulse output (PTO) to provide a specified pulse number of square wave output [2]. The frequency (or period) of the output pulse is linearly varied during acceleration and deceleration, while the constant frequency section remains constant. Once the specified number of pulses are generated, the PTO output becomes low, and the pulse is generated until a new specified value is loaded.

\subsection{Door Operation Obstacle Detection, Alarm.}

Set up a sensor to detect a man or an object in the middle of the door during the closing of the door. The active type infrared anti pinch sensor is composed of an infrared emitter and a receiver which are emitted at the frequency of [3], and the infrared frequency is different from the nature and the human body. During the closing process, the receiver receives no light signal to generate a negative pulse, and the pulse is used as an interrupt signal of PLC, and the PLC controls the door body to move in the opposite direction. This is a very important protective function in preventing the collision of people or the same time, the protection of automatic door motor not due to overload and burn.

\subsection{Host of PLC.}

In the control circuit, The input control signals, a total of 11 points in the PLC, including the human body induction signal detector 2 , automatic door operation position detection signal, gate movement obstacle detection signals a (combined with the characteristic of PLC, the signal in the circuit to convert to switch contact signal), and manual door open button signal, manual door closing button signal, door start signal button, the door will stop button signal, a thermal relay auxiliary normally closed contact signal. PLC output of the control signal points to 4 points, including the stepper motor control signal 2 points, 2 points of the gate operating obstacle alarm signal.

\section{Software Design}

\subsection{Main Program Module.}

First of all, the system initialization, including the opening and closing process of the various stages of the variable initialization, interrupt settings, etc., and then PLC according to the input signal for different processing. When the stop button is pressed, the PLC does not respond to the closing request, and the motor stops working, and the door is still not moving. When press the start button, the PLC begins response open and close requests: if press the manual button to open the door, open call subroutine; if the press manual door closing button, call closed subroutine; if you receive the induction signal of human body, first call door subroutine, after confirming that no one, after a period of time delay, call closed subroutine. The main program flow chart is shown in figure1. 


\subsection{Open Subroutine and Closed Subroutine Module.}

The flow chart of the procedure for opening the door is shown in figure 2. When opening the door, first look at the door closed limit signal is valid, if effective, the door is completely closed, the trip variable clearance 0. Because PLC re execute the program, so the detection manually open the door or a human body induction signal in the effective after the initial call to open the door subroutine. If you set the PLC high speed pulse output parameters to prepare for a drive motor. And then detect the motor running, turn a circle of travel variables plus one. The last door detection limit, closing signal output of the motor, to avoid when the door is fully open, motor movement.

Closed subroutine flow chart as shown in figure 3. When closing the door, the first detection manual door closing or the human body induction signal is the first time to call the closed subroutine, if you set the PLC high speed pulse output parameters, the output motor drive signal, the motor reverse. And then detect the operation of the motor, to reduce the transfer of a circle of travel variables. In the end, it is effective to detect the door closing limit. If the door is closed, the motor signal is cut off. Note that each open (closed) door, the door is not necessarily completely closed (open), with the trip variable to characterize the position of the electric door. High speed pulse output function can be based on the size of the stroke of the output of the corresponding pulse, so that the motor according to the acceleration, constant speed, deceleration of the way to run.

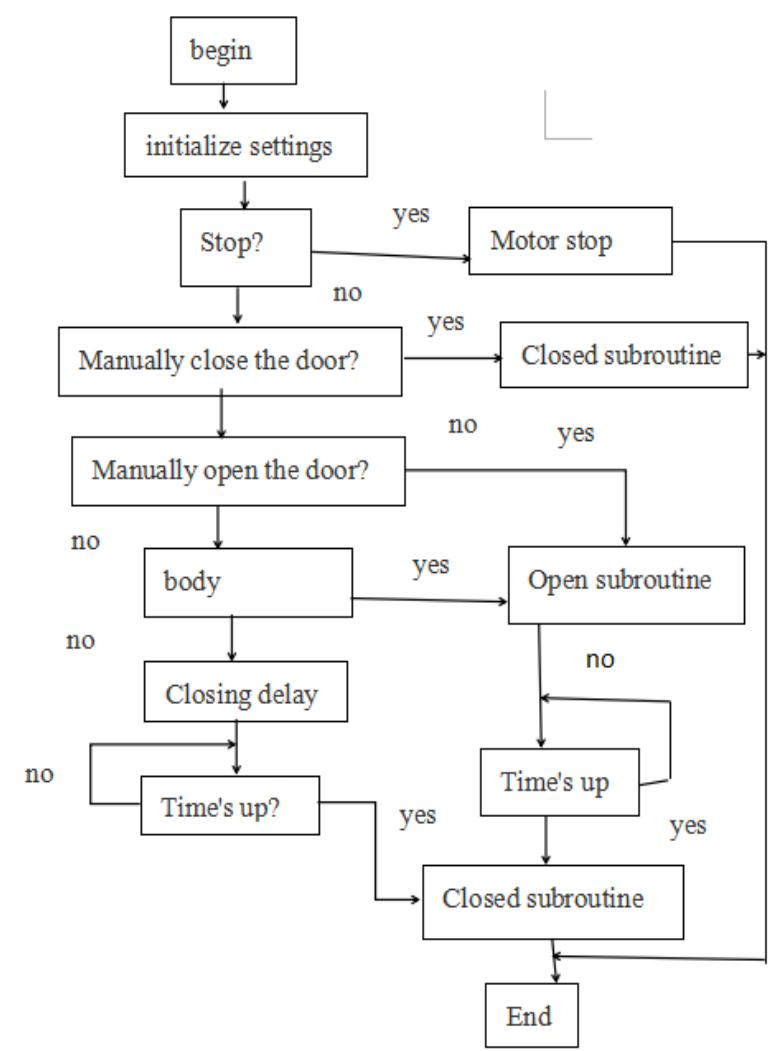

Fig.1 Main program flow chart

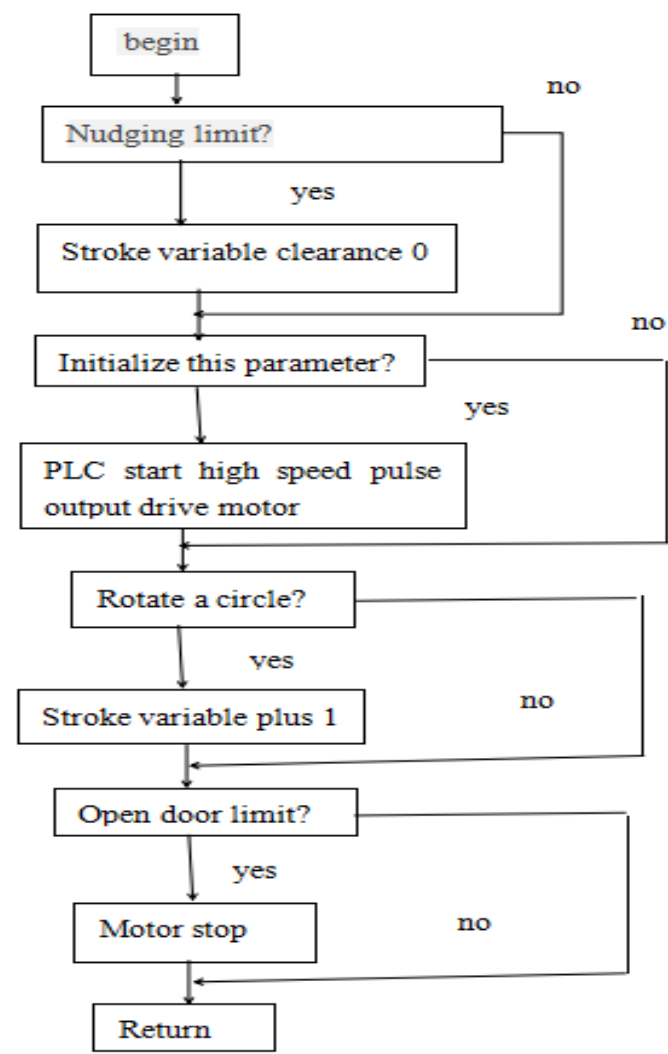

Fig.2 Open subroutine flow chart 


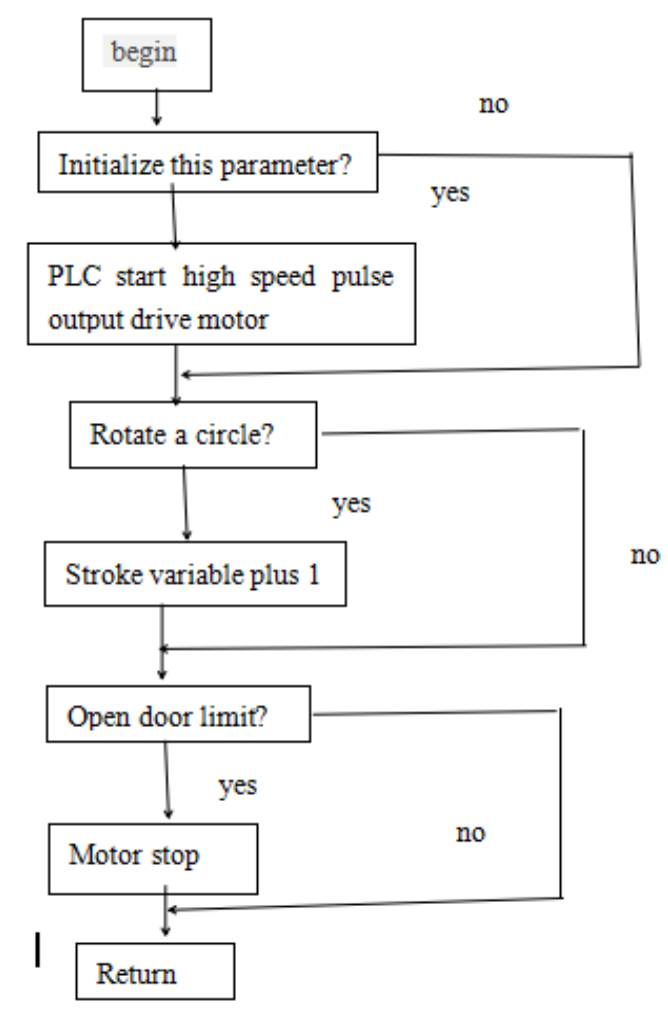

Fig.3 Closed subroutine flow chart

\section{Summary}

This research applies PLC to automatic door control system. Points to play PLC: high reliability and anti-interference characteristics, external wiring Simple, flexible, easy to maintain. Double component type pyroelectric red external sensing. The infrared photoelectric sensor is used for detecting the human body in and out of the situation. The sensor is sensitive and reliable, and is not affected by the ring. The sensor is sensitive and reliable, and is not affected by environmental factors. The system has been debugged by hardware and software, which has been applied in practice, and has high reliability, flexibility and economy.

\section{References}

[1]. Zhi ming Wang. Method of achieving analog value output based on PLC digital output. Mechanical\& Electrical Engineering Magazine. Vol. 26 (2009) No. 05, p. 105-108.

[2]. Hui Li, Hai xia Li, Xian ying Feng. Mobile robot s control system based on MCU\&CPLD. Mechanical\& Electrical Engineering Magazine. Vol. 26 (2009) No. 08, p. 100-104.

[3]. Yan ping Guo. Electrical control and PLC. Beijing Normal University Publishing, 2009, p. 264-275. 